Mercadé Aloy, J., Magrinyà Torner, F., Cervera Alonso de Medina, M. (2018): "Descifrando la forma urbana: un análisis de patrones de agrupamiento basado en SIG", GeoFocus (Artículos), no 22, p. 3-19. ISSN: $1578-5157$ http://dx.doi.org/10.21138/GF.612

\title{
DESCIFRANDO LA FORMA URBANA: UN ANÁLISIS DE PATRONES DE AGRUPAMIENTO BASADO EN SIG
}

\author{
JOSEP MERCADÉ ALOY ${ }^{1}$, FRANCESC MAGRINYÀ TORNER ${ }^{1}$, MARINA CERVERA \\ ALONSO DE MEDINA ${ }^{2}$ \\ ${ }^{1}$ Departament d'Enginyeria Civil i Ambiental. Universitat Politècnica de Catalunya \\ C. Jordi Girona, 1-3. 08034 Barcelona, España \\ josep.mercade@upc.edu, francesc.magrinya@upc.edu \\ ${ }^{2}$ Departament d'Urbanisme i Ordenació del Territori. Universitat Politècnica de Catalunya \\ Campus Sud Avgda. Diagonal 649. 08028 Barcelona, España \\ marina.cervera@upc.edu
}

\section{RESUMEN}

Este artículo propone un método de clasificación de tejidos urbanos a partir de un conjunto de variables derivadas de su propia morfología -edificios, parcelas, manzanas y calles-, centrado en el ámbito del Área Metropolitana de Barcelona.

Se introducen atributos de densidad en relación a la condición de alineación de vial y la presencia de medianeras que complementan los parámetros más comunes en torno al techo y la huella construida. Se utiliza el algoritmo k-means para construir un conjunto de grupos homogéneos.

Los resultados revelan un mosaico de tejidos urbanos nítido, compuesto de 12 grupos disimilares. Se sugiere la futura combinación de los valores de forma obtenidos con otros parámetros no estrictamente físicos -configuración socioeconómica, energía y equidad, entre otrospara evaluar los efectos de la morfología urbana en cada caso.

Palabras clave: forma urbana, morfología urbana, análisis de clústeres, SIG

\section{DECHIPERING URBAN FORM: A GIS-BASED CLUSTER ANALYSIS}

\section{ABSTRACT}

This paper contributes to the classification of urban form on the basis of variables arising from its morphology-buildings, plots, blocks and streets-, taking the case of the Metropolitan Area of Barcelona as a case study. 
Mercadé Aloy, J., Magrinyà Torner, F., Cervera Alonso de Medina, M. (2018): "Descifrando la forma urbana: un análisis de patrones de agrupamiento basado en SIG", GeoFocus (Artículos), no 22, p. 3-19. ISSN: $1578-5157$ http://dx.doi.org/10.21138/GF.612

New density attributes regarding street alignment and party walls are introduced together with the most common floor and ground surface indexes. The algorithm k-means is used to perform a cluster analysis.

The results reveal a clear mosaic of urban fabrics, composed of 12 dissimilar groups. The combination of the outcomes with other non-physical parameters-socio-economic indicators, energy and equity, among others-is suggested with a view to evaluate urban form effects.

Keywords: urban form, urban morphology, clustering analysis, GIS.

\section{Introducción}

La morfología urbana se encarga de estudiar los resultados tangibles -forma- de los procesos de transformación de la ciudad como consecuencia de la acción de fuerzas culturales, económicas y sociales (Moudon, 1997). La caracterización dibujada de los tejidos urbanos cuenta con una larga tradición como confirman los encuentros del International Seminar on Urban Form (para una revisión del caso español ver p.e. Vilagrasa, 1998).

El interés de estas aproximaciones radica en la posibilidad de correlacionar la configuración espacial de la ciudad con sus fortalezas y fragilidades para orientar el planeamiento consecuentemente. Talen (2011) pone de manifiesto el potencial de una lectura basada en parámetros derivados de la forma urbana para evaluar configuraciones urbanas actuales y poder proponer elementos de transformación hacia entornos construidos más sostenibles. En este mismo sentido, Marshall (2005) señala la necesidad de analizar los patrones urbanos existentes y de plantear los del futuro en el marco del concepto de sostenibilidad. Ello implica necesariamente el testeo de variables relacionadas con la forma urbana para comprobar sus efectos.

Si bien existen múltiples indicadores de la sostenibilidad, tales como la contaminación atmosférica o los kilómetros viajados en vehículo privado, no existe un consenso tan amplio en torno a la identificación de patrones de la configuración espacial y relacional urbana (Stead y Marshall, 2001).

Sin embargo, la producción científica pone de manifiesto el tanteo de múltiples variables de la forma como base para establecer áreas homogéneas de la ciudad en términos de patrones de configuración espacial (ver p.e. Berghauser y Haupt, 2007), que frecuentemente van de la mano del análisis de clústeres que automatiza dichas clasificaciones (ver p.e. Schrimer y Axhausen, 2015). Se trata, pues, de la introducción de métodos cuantitativos dirigidos a descifrar los vectores que construyen la forma urbana y testar su validez usándolos como variables descriptivas de paquetes de ciudad homogéneos.

En este contexto, se propone realizar un análisis de agrupamiento mediante SIG a partir de un conjunto de variables explicativas de los tejidos urbanos del Área Metropolitana de Barcelona, mediante métodos cuantitativos, con el propósito de servir como base para estudios urbanos de todo tipo.

\section{Estado de la cuestión}

El origen de los estudios de tipomorfología se sitúa en torno a dos investigadores principales. Se trata del geógrafo alemán Conzen que emigró a Inglaterra después de la segunda 
Mercadé Aloy, J., Magrinyà Torner, F., Cervera Alonso de Medina, M. (2018): "Descifrando la forma urbana: un análisis de patrones de agrupamiento basado en SIG", GeoFocus (Artículos), no 22, p. 3-19. ISSN: $1578-5157$ http://dx.doi.org/10.21138/GF.612

Guerra Mundial y del arquitecto italiano Muratori. En el primer caso Whitehand dio continuidad a los estudios de Conzen formando el Urban Morphology Research Group en la Universidad de Birmingham y, en el segundo caso, Caniggia extendió la influencia de la tradición italiana. Todavía una tercera escuela emergió en Francia a finales de los años 1960s de la mano de los arquitectos Phillipe Panerai y Jean Castex, conjuntamente con el sociólogo Jean-Charles Castex DePaule. Con el tiempo establecieron contacto con investigadores italianos, españoles y de America Latina (Moudon, 1997).

La evolución de una lectura esencialmente descriptiva -dibujada con métodos no cuantitativos- del territorio de la ciudad, de raíz morfotipológica, ha dado múltiples frutos a considerar. En el caso europeo son destacables los estudios de la Región de Milán dirigidos por Boeri et al. (1993), las contribuciones de Solà-Morales (1979, 1989, 1993) y más recientemente las de Font et al. (1999) con La Construcció del territori metropolità : morfogènesi de la regió urbana de Barcelona, así como Patrons urbanístics de les activitats econòmiques : Regió Metropolitana de Barcelona (Font, 2012). De la misma manera, deben mencionarse ciertos trabajos de compilación tales como Le Transformazioni dell'Habitat Urbano, coordinado por Secchi (1993), o La Explosión de la ciudad: transformaciones territoriales en las regiones urbanas de la Europa Meridional de Font et al. (2004), que explora la evolución del último cuarto de siglo XX para las regiones de Portugal, Francia, Itàlia y España: Lisboa, Porto, Marsella, Montpellier, Boloña, Génova, Milán, Nápoles, el Véneto central, Barcelona, Donostia-Bayona, Madrid y Valencia.

Paralelamente a dicha evolución y decantación de una tradición desde una urbanística descriptiva existe la inevitable tendencia a la introducción de métodos de raíz cuantitativa. Giles et al. (2012) han puesto de relieve la pertinencia del uso de la tecnología informática para la realización de estudios de morfología urbana y Schrimer y Axhausen (2015) han evidenciado la necesidad de identificar un conjunto de atributos que puedan caracterizar la forma urbana en este sentido. La definición de un conjunto de variables descriptivas de los tejidos resulta esencial para el objetivo de esta investigación: la identificación cuantificada de tejidos urbanos homogéneos.

La producción científica y la literatura especializada en general permiten identificar múltiples contribuciones en relación a la identificación de patrones, variables descriptivas de la forma urbana, el rol de la red de espacios públicos de soporte de los tejidos y el uso de métodos de obtención de clústeres (Schrimer y Axhausen, 2015; Gil et al., 2012).

Es especialmente relevante la proposición de patrones para ciudades, barrios y edificios como lenguaje e instrumento para los procesos de diseño a distintas escalas por parte de Alexander et al. (1977).

En cuanto a parámetros descriptivos de la forma urbana, Berghauser y Haupt (2007) ponen de relieve el papel de la densidad como variable indispensable para la caracterización de los tejidos urbanos, proponiendo una matriz -spacemate- que recoge el índice de edificabilidad en el eje vertical, el parámetro de ocupación en el eje horizontal -porcentaje que supone la huella edificaday las variables derivadas: espacio libre sin edificar y el número de plantas. Aquellos tejidos urbanos que comparten valores similares para dichas variables tienden a agruparse en ciertas zonas de la matriz spacemate. García (2016) introduce una variante de dicha matriz -citymate - en la cual sitúa las ciudades españolas en base a su densidad en habitantes por hectárea y ocupación en porcentaje. Morganti et al. (2012) exploran la relación entre morfología urbana y comportamiento energético y ambiental, tomando como referencia la matriz spacemate. Meinel et al. (2008) proponen una clasificación de edificios y manzanas usando atributos como su proyección horizontal. Haggag y 
Mercadé Aloy, J., Magrinyà Torner, F., Cervera Alonso de Medina, M. (2018): "Descifrando la forma urbana: un análisis de patrones de agrupamiento basado en SIG", GeoFocus (Artículos), no 22, p. 3-19. ISSN: $1578-5157$ http://dx.doi.org/10.21138/GF.612

Ayad (2002) abordan la identificación de áreas estructurales homogéneas de la ciudad cuantificando la cubiertas del suelo, el entorno construido, los usos del suelo, la edad de los edificios y la densidad de las áreas urbanas. Laskari et al. (2008) han investigado hasta que punto los atributos cuantificables del espacio pueden dar cuenta de la identidad espacial del entorno construido, tomando la manzana como unidad de estudio. Dillenburger (2010) propone una herramienta que permite identificar edificios morfológicamente parecidos al seleccionado por el usuario.

El rol de la topología de la red viaria es especialmente presente en las aproximaciones en torno a las teorías Space Syntax que, entre otras cosas, han propuesto algoritmos para analizar la configuración espacial de un grafo de calles, dando cuenta de su relación con los patrones de localización de actividad. Las referencias más relevantes en esta materia pueden encontrarse en los libros de Hillier and Hanson (1984), Hillier (1996) y Hanson (1998), así como en los proceedings de los Space Syntax Simposia. Sevtusk y Mekonnen (2012) han propuesto la extensión del uso de dichas medidas de centralidad usando los edificios y sus atributos -techo, usos, número de residentes, lugares de trabajo, altura entre otros- como orígenes y destinos de la caracterización topológica de la forma urbana. Cardozo et al. (2010) proponen una lectura de la morfología urbana que considera las variables de población y empleo en un radio de 1.500 metros -se miden los recorridos sobre la malla de calles-alrededor de las estaciones de la red de metro de Madrid, con la finalidad de establecer correlaciones con la entradas totales a dichas estaciones. Ye y Nes (2014) combinan los métodos Space Syntax con la matriz spacemate y el índice Mixed Use Index (MXI) desarrollado por Van den Hoek (2009) para medir el grado de madurez de los procesos urbanos. Oliveira (2012), propone la metodología morpho que combina siete variables: accesibilidad de las calles a partir de medidas de centralidad, densidad de parcelas, año de construcción de los edificios, dimensión de las manzanas, alineación de los edificios, la proporción entre altura de los edificios y ancho de calle, y los usos de los edificios.

La proliferación de estudios que introducen los análisis de agrupamiento es, también, un hecho constatable. Thomas et al. (2010) utilizan la dimensión fractal y las curvas de comportamiento escalar para caracterizar las áreas urbanas a partir de la formación de clústeres $k$ medoid, demostrando que paquetes de ciudad con formas de crecimiento similares se agrupan conjuntamente. Hamaina et al. (2012) realizan una caracterización de la forma urbana y del espacio libre usando como único dato la huella de las edificaciones, para un análisis de clústeres a partir de self-organized maps. Gil et al. (2012) utilizan el algoritmo k-means para definir clústeres de manzana y de segmentos de red viaria -calles- a partir de variables de geometría, densidad, forma, uso del espacio público y topología de la red viaria. En el caso de las manzanas destacan los parámetros de ordenación básicos y en el caso de las calles se combina el ancho de calle y su proporción dedicada al peatón con las medidas de centralidad closeness y betwenness. Schrimer y Axhausen (2015) plantean un análisis de grupos basado en algoritmo k-means para distintas escalas: el tejido urbano, el distrito y el municipio. Hecht et al. (2013) proponen la clasificación automática de las huellas de los edificios a partir del reconocimiento de patrones y machine learning.

Con todo, se perfila la pertinencia de un conjunto de aproximaciones dedicadas a la identificación de variables descriptivas de los tejidos urbanos y el subsiguiente análisis de clústeres. La contribución de este artículo será, pues, la proposición de un conjunto de atributos de la forma urbana y la identificación de grupos homogéneos como herramienta de análisis de la ciudad. 


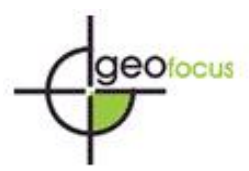

Mercadé Aloy, J., Magrinyà Torner, F., Cervera Alonso de Medina, M. (2018): "Descifrando la forma urbana: un análisis de patrones de agrupamiento basado en SIG", GeoFocus (Artículos), no 22, p. 3-19. ISSN: $1578-5157$ http://dx.doi.org/10.21138/GF.612

\section{Materiales, datos y métodos}

\subsection{El caso de estudio.}

El caso de estudio propuesto es el Área Metropolitana de Barcelona, integrada por 36 municipios con una población en torno a los tres millones de habitantes (Área Metropolitana de Barcelona, n.d.). El $48 \%$ del suelo del ámbito se encuentra ocupado y, por lo tanto, se dispone de un gran abanico de configuraciones urbanas para analizar. El estudio que se presenta explora únicamente la situación actual vigente a partir de los datos disponibles más actualizados.

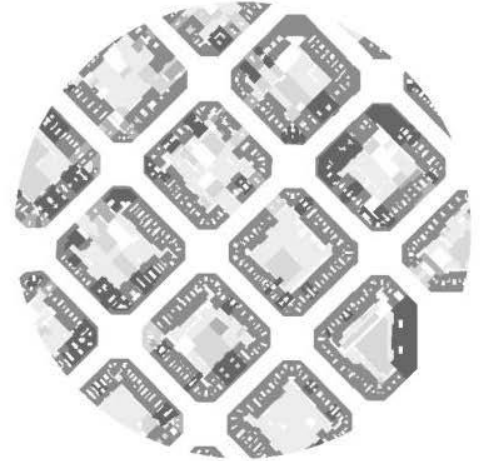

(a)

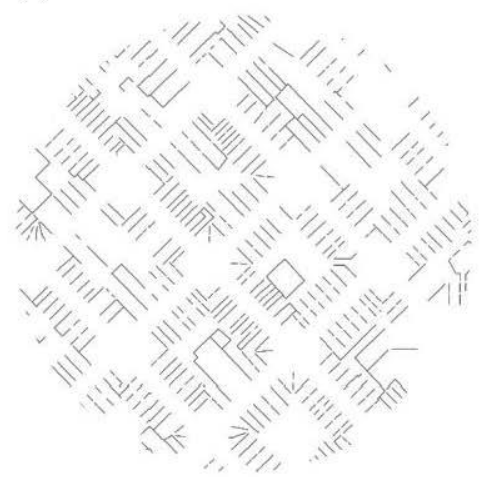

(d)

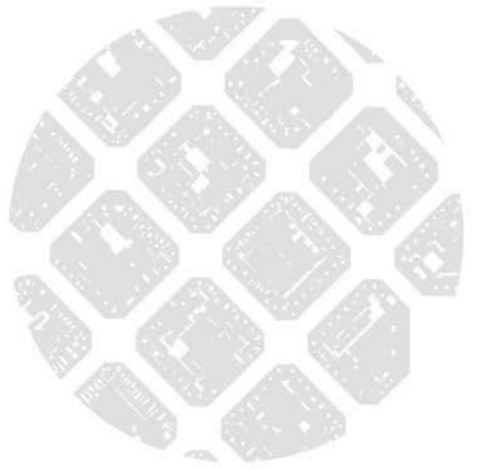

(b)

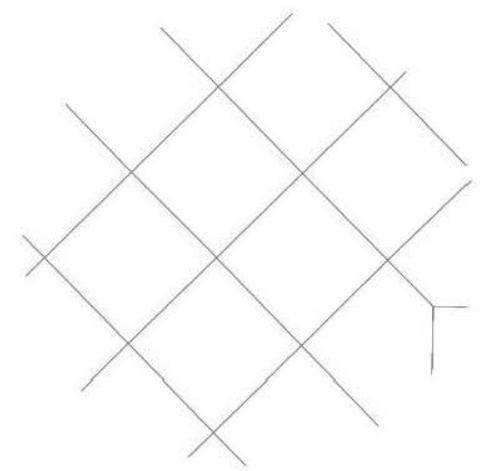

(e)

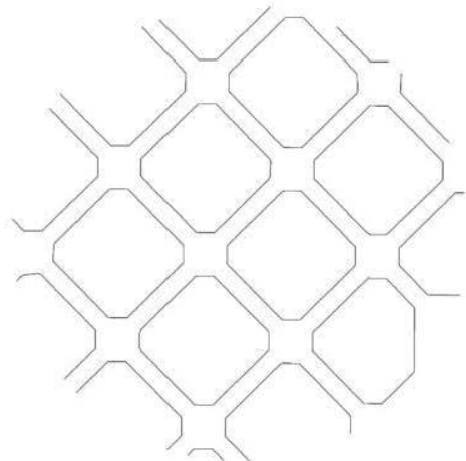

(c)

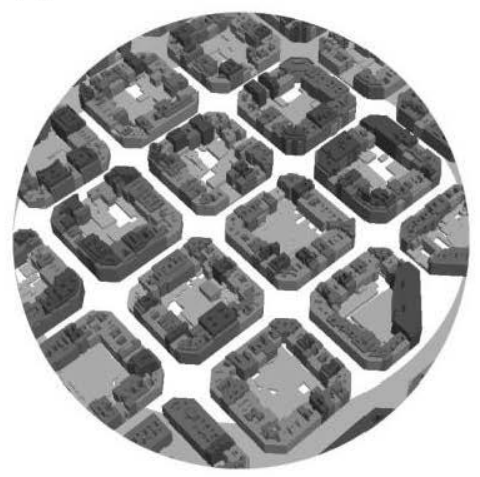

(f)

Figura 1. Variables descriptivas: (a) edificabilidad bruta, (b) ocupación, (c) densidad de alineación a vial, (d) densidad de medianeras, (e) densidad de red viaria y (f) Compacidad. Fte.: Elaboración propia a partir de datos catastrales 2017. Dirección General del Catastro.

\subsection{Los datos.}

Para definir el marco espacial de referencia del caso de estudio se utiliza la Base Municipal de Catalunya vigente y la Base de Datos Geoespacial de los Polígonos Industriales de Catalunya, elaboradas por el Instituto Cartográfico y Geológico de Catalunya (ICGC).

Para obtener los valores de las variables que intervienen en la caracterización de los tejidos urbanos se parte de la cartografía urbana vectorial de la Dirección General del Catastro que se puede obtener en la Sede Electrónica del Catastro (SEC). Concretamente, se descargan los datos 
Mercadé Aloy, J., Magrinyà Torner, F., Cervera Alonso de Medina, M. (2018): "Descifrando la forma urbana: un análisis de patrones de agrupamiento basado en SIG", GeoFocus (Artículos), no 22, p. 3-19. ISSN: $1578-5157$ http://dx.doi.org/10.21138/GF.612

asociados a: las subparcelas urbanas que representan los volúmenes edificados dentro de una parcela, las parcelas catastrales, las agrupaciones de parcelas catastrales -manzanas de urbana- y los ejes de elementos lineales -carreteras y calles. La geoinformación se obtiene para cada municipio que forma parte del Área Metropolitana de Barcelona.

El conjunto de dicha geoinformación encapsula la esencia de la forma urbana y permite obtener los valores que toman las variables para cada ubicación deseada. A continuación se describen sucintamente las variables intervinientes.

Un primer paquete de variables, utilizado por Berghauser y Haupt (2007) como pilares del concepto spacemate son el índice de edificabilidad bruta y la ocupación. En realidad se trata de parámetros de ordenación ampliamente utilizados en el planeamiento urbanístico (ver p.e. Esteban, 1981). El índice de edificabilidad bruta se obtiene mediante el cociente entre el techo edificable -en nuestro caso el techo ya construido- y la superficie del área considerada, incluyendo las calles y otros espacios libres. La ocupación es el cociente entre la superficie ocupada por la proyección en planta de las partes edificadas y la superficie de la parcela que las contiene. En nuestro caso se utilizará en su forma bruta, es decir para áreas de ciudad que incluyen calles y otros espacios libres.

Por otro lado se ha considerado imprescindible recoger ciertas relaciones de contacto perimetral entre la edificación y su parcela que dan cuenta del sistema de ordenación en el que se encuentran. Se propone, en primer lugar, calcular la densidad de alineación a vial como el cociente entre la longitud de fachada en contacto con el vial -intersección entre los frentes de parcela y la edificación- y el área bruta de ciudad considerada. En segundo lugar, se calcula la densidad de medianeras como el cociente entre la longitud de pared medianera de más de una planta intersección entre los laterales y fondos de parcela con la edificación- y el área bruta considerada.

Complementariamente, se propone trabajar con la variable compacidad que se define como el cociente entre el volumen edificado y la superficie de su envolvente.

Finalmente, se considera la densidad de red viaria como cociente entre la longitud de los ejes de calle presentes en un determinado ámbito y su área bruta (véase figura 1).

\subsection{Técnicas de análisis.}

Las técnicas de análisis que se utilizaran centran su atención en dos aspectos esenciales: cómo capturar los valores asociados a las variables que caracterizan los tejidos urbanos y cómo establecer las áreas homogéneas de ciudad.

En el primer caso, resulta imprescindible considerar los tejidos urbanos como paquetes de ciudad, incluyendo, por lo tanto, sus calles y otros espacios libres. En este sentido, se abandona la inmediatez de un análisis desde la unidad básica -edificación, parcela o manzana- puesto que se perdería totalmente el sentido de tejido: el conjunto de edificaciones que soportan los agrupamientos de parcelas que, a su vez, se organizan en manzanas que se estructuran a partir de la red viaria. Ello implica la necesidad de definir una unidad mínima de área bruta sobre la cual calcular las variables intervinientes.

Se propone construir una malla ortogonal de círculos de radio 250 metros, cuyos centros equidistan 250 metros entre ellos. Dichos círculos se sobreponen un máximo de $250 \mathrm{~m}$ (véase figura 2). De esta forma se pueden calcular los valores de las variables referidos al área de cada círculo y 


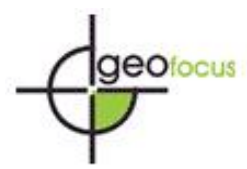

Mercadé Aloy, J., Magrinyà Torner, F., Cervera Alonso de Medina, M. (2018): "Descifrando la forma urbana: un análisis de patrones de agrupamiento basado en SIG", GeoFocus (Artículos), no 22, p. 3-19. ISSN: 1578-5157 http://dx.doi.org/10.21138/GF.612

utilizar sus centros para visualizar los datos obtenidos. La superposición entre círculos permite capturar la transición entre unos tejidos y otros de manera suavizada.

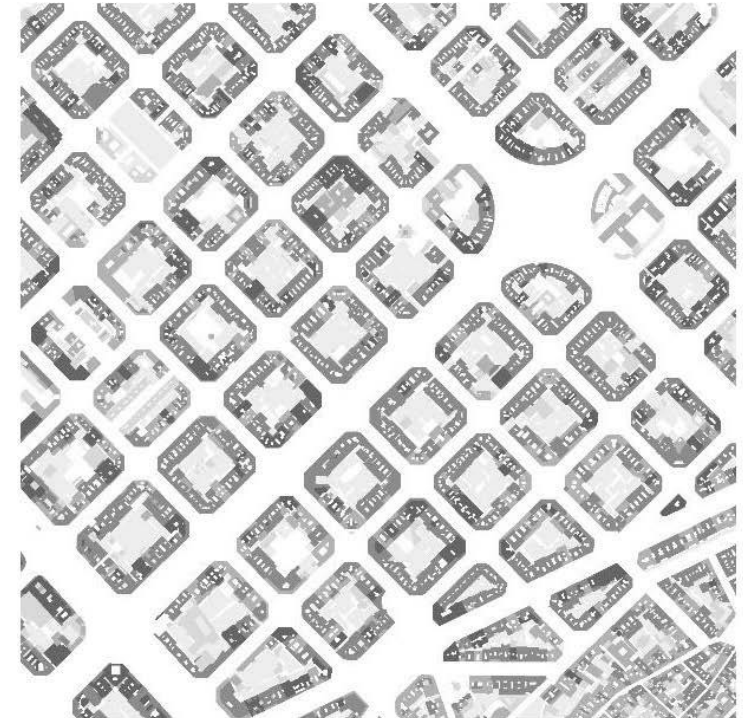

(a)

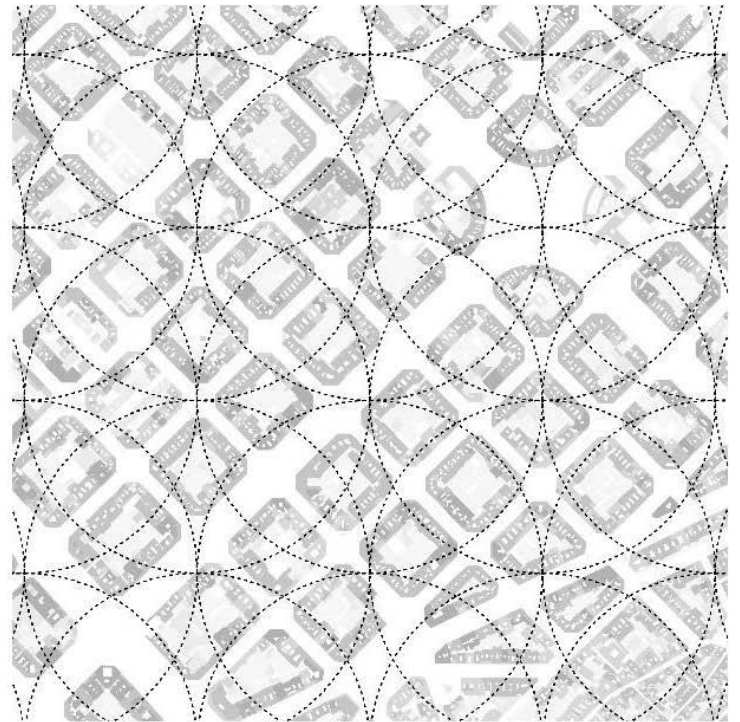

(b)

Figura 2. Captura de datos de forma urbana: (a) tejido urbano del ensanche de Barcelona, (b) círculos de $250 \mathrm{~m}$ de radio.

Fte.: Elaboración propia a partir de datos catastrales 2017. Dirección General del Catastro.

El propio planteamiento hace necesario corregir aquellos círculos que no se encuentran totalmente embebidos en el medio urbano objeto de estudio. En nuestro caso se quiere centrar la atención exclusivamente en los tejidos que contienen el ingrediente vivienda. En consecuencia, pues, se descartan los círculos que intersectan polígonos industriales y se considera su superficie parcialmente en aquellos casos en los cuales se encuentran a caballo entre el medio urbano -en el sentido catastral- y el rústico.

En el segundo caso se trata de establecer las áreas homogéneas a partir del análisis de grupos que ha de permitir separar conjuntos de elementos que sean tan diferentes entre ellos como sea posible. En nuestro caso interesa obtener los grupos sin considerar restricciones espaciales -sin imponer que los grupos se formen por razones de contigüidad- dado que se espera que las tipologías de tejidos urbanos se repitan en los distintos núcleos urbanos. El software que se utiliza (ArcMap 10.5) permite calcular dichos grupos a partir del algoritmo $k$-means.

Jain (2010) realiza una revisión exhaustiva de los métodos de agrupamiento más destacados, situando el origen del algoritmo k-means, ampliamente utilizado hoy en día por ser el más simple y popular, 50 años atrás. Si bien la terminología $k$-means fue utilizada por primera vez por MacQueen (1967), la idea se atribuye a Steinhaus (1957), mientras que el algoritmo estandarizado fue propuesto por Lloyd (propuesto en 1957, publicado en 1982)

La presentación del problema se plantea a partir de un conjunto de observaciones $\left(\mathbf{x}_{1}\right.$, $\left.\mathbf{x}_{2}, \ldots, \mathbf{x}_{\mathrm{n}}\right)$, donde cada observación es un vector real de $\mathrm{d}$ dimensiones y $k$-means construye una partición de las observaciones en $\mathrm{k}$ conjuntos $(\mathrm{k} \leq \mathrm{n})$ con el fin de minimizar la suma de los cuadrados dentro de cada grupo $\mathbf{S}=\left\{\mathrm{S}_{1}, \mathrm{~S}_{2}, \ldots, \mathrm{S}_{\mathrm{k}}\right\}$ : 
Mercadé Aloy, J., Magrinyà Torner, F., Cervera Alonso de Medina, M. (2018): "Descifrando la forma urbana: un análisis de patrones de agrupamiento basado en SIG", GeoFocus (Artículos), no 22, p. 3-19. ISSN: $1578-5157$ http://dx.doi.org/10.21138/GF.612

Donde $\mu_{\mathrm{i}}$ es la media de puntos en $\mathrm{S}_{\mathrm{i}}$.

$$
\arg \min \sum_{i=1}^{k} \sum_{x_{j} \in S_{i}}\left\|x_{j}-\mu_{i}\right\|^{2}
$$

El algoritmo k-means necesita forzosamente iniciarse con un número de grupos inicial prefijado. El error como consecuencia de la suma de los cuadrados se reduce al aumentar el número de grupos $\mathrm{y}$, por lo tanto, únicamente puede ser minimizado para una número de grupos determinado (Jain, 2010). El algoritmo necesita identificar primero los elementos semilla para hacer crecer cada grupo. La primera semilla se selecciona aleatoriamente. A continuación se asignan todos los elementos a la entidad de semilla más cercana en el espacio de datos. Para cada grupo de elementos se calcula un centro medio de los datos y se vuelve a asignar cada elemento al centro más cercano. El proceso de obtener un centro medio de datos para cada grupo y reasignar los elementos a dicho centro más cercano continúa iterativamente hasta que se estabiliza la pertenencia a cada grupo. El software utilizado permite obtener un informe en el cual se incluye, entre otras cosas, el valor $\mathrm{R}^{2}$ para cada variable específica (ESRI, n.d.).

En consecuencia, es necesario establecer un número de grupos que tenga sentido e incluir una cantidad de variables de discriminación que sean representativas. En nuestro caso podemos resolver claramente el primer dilema y encarar el segundo en función de los resultados, planteando la posible eliminación de alguna variable si se estimara oportuno.

Se necesita distinguir entre un mínimo de 4 grandes grupos que, a su vez, deberíamos ser capaces de graduar y matizar internamente: tejidos históricos, ordenaciones compactas generalmente en manzana cerrada, ordenaciones en volumetría específica en manzana abierta y paquetes de baja densidad. Se considera que los tres últimos grupos deberían poderse matizar e incluso prever situaciones híbridas. Con todo ello, se propone distinguir entre 12 grupos.

\section{Descripción y análisis de resultados.}

El primer paso consiste en calcular el valor de las variables consideradas para los círculos de 250 metros de radio que se han propuesto como método de captura de los parámetros de forma urbana. Los resultados se visualizan en el centro de cada círculo, como cantidades graduadas tamaño del punto-, agrupando los valores en cinco rangos obtenidos mediante el método de grupos naturales (Jenks, 1967) y su posterior redondeo manual (véase figura 3). El índice de edificabilidad bruta sitúa claramente la masa crítica de la ciudad sin ser capaz de identificar los tejidos más allá de resaltar los centros y subcentros, así como los paquetes de baja densidad. La ocupación permite obtener de forma graduada la presencia de espacios libres en los tejidos analizados. El resultado obtenido con la densidad de alineación a vial es especialmente útil para identificar los núcleos históricos y los tejidos suburbanos. La densidad de medianeras afina el caso anterior, poniendo de manifiesto las intensidades de los tejidos de grano más fino, de mayor intensidad parcelaria. La compacidad informa puramente sobre la forma preponderante de los edificios en las manzanas contenidas en los círculos de $250 \mathrm{~m}$ propuestos. Si bien su lectura aislada dificulta la observación de patrones nítidos -edificios muy disimilares pueden compartir valores similares de compacidad-, se considera que necesariamente se trata de una variable que debería contribuir a matizar la naturaleza de los tejidos urbanos en combinación con las restantes. Finalmente, la densidad de red viaria ofrece un paisaje de datos más confuso si se quiere interpretar de forma aislada. 
Mercadé Aloy, J., Magrinyà Torner, F., Cervera Alonso de Medina, M. (2018): "Descifrando la forma urbana: un análisis de patrones de agrupamiento basado en SIG", GeoFocus (Artículos), no 22, p. 3-19. ISSN: 1578-5157 http://dx.doi.org/10.21138/GF.612

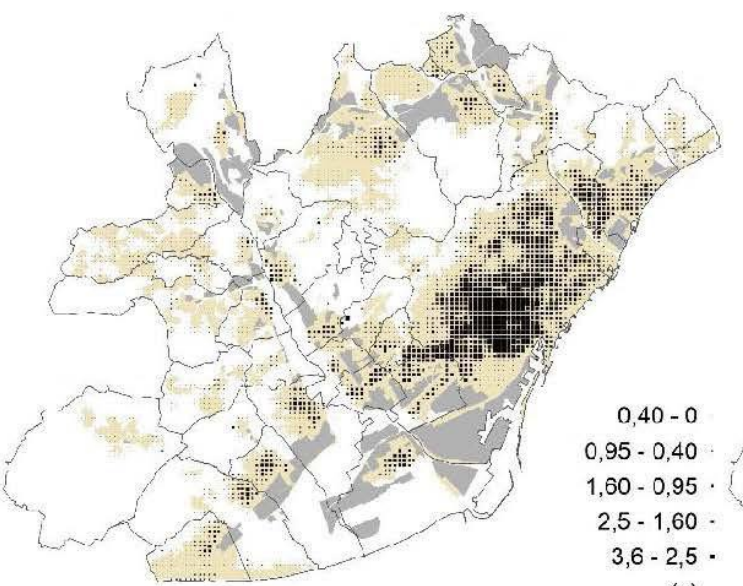

(a)

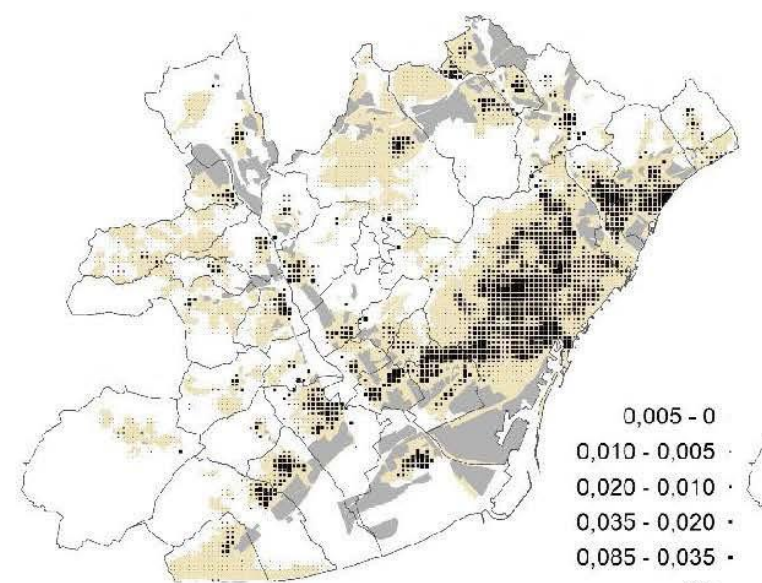

(c)

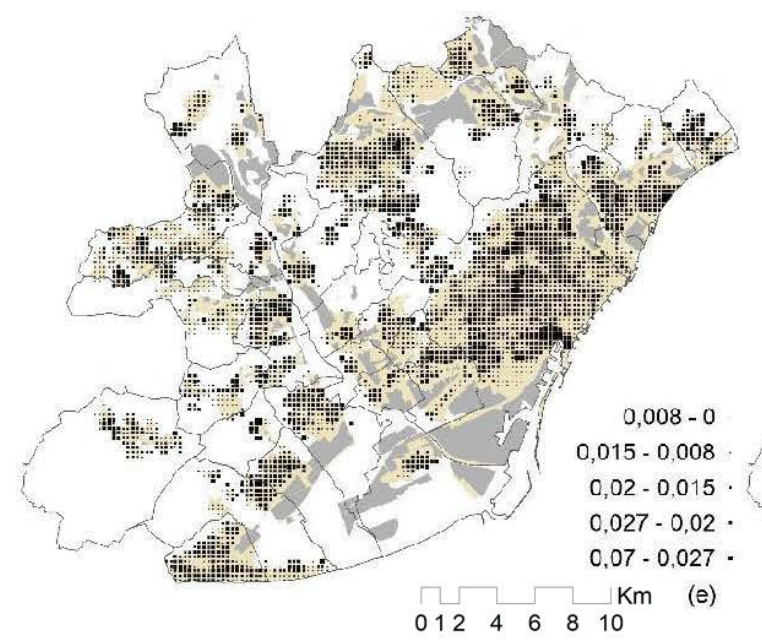

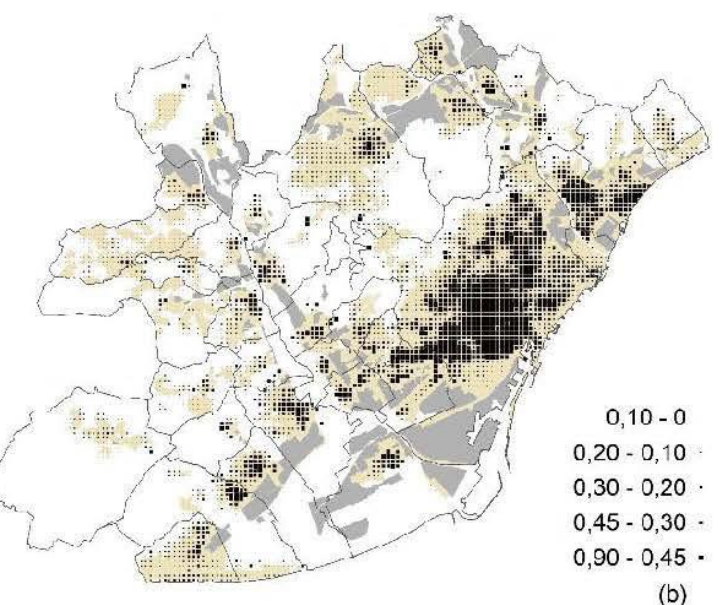

(b)

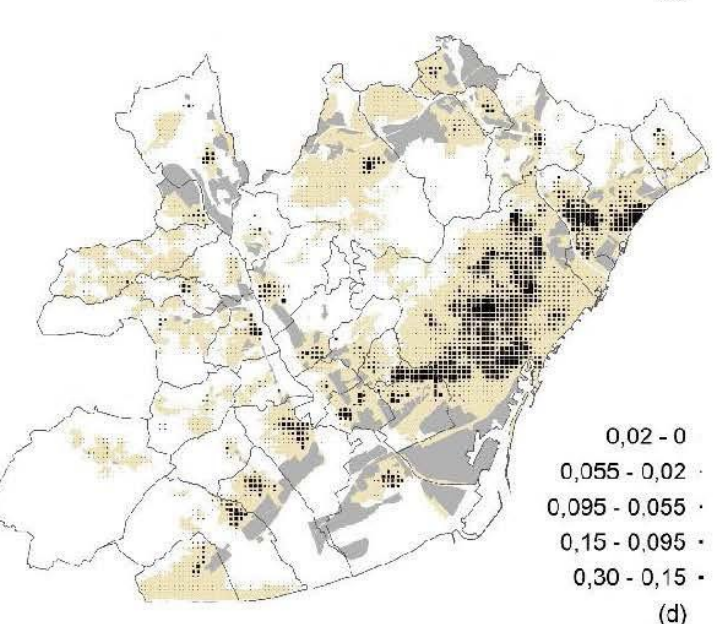

(d)

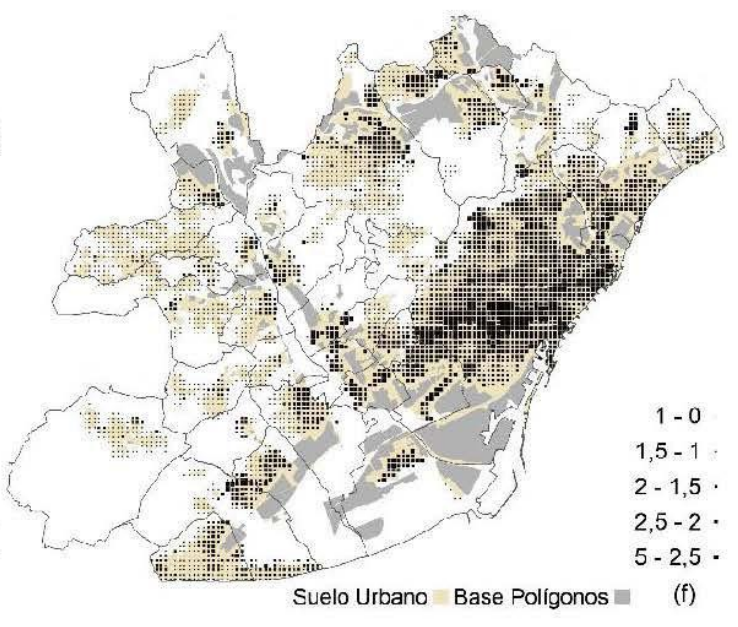

Figura 3. Resultados: (a) edificabilidad bruta, (b) ocupación, (c) densidad de alineación a vial, (d) densidad de medianeras, (e) densidad de red viaria y (f) Compacidad.

Fte.: Elaboración propia a partir de datos catastrales 2017. Dirección General del Catastro. 
Mercadé Aloy, J., Magrinyà Torner, F., Cervera Alonso de Medina, M. (2018): "Descifrando la forma urbana: un análisis de patrones de agrupamiento basado en SIG", GeoFocus (Artículos), no 22, p. 3-19. ISSN: $1578-5157$ http://dx.doi.org/10.21138/GF.612

Una vez se dispone de las variables intervinientes es necesario establecer su grado de contribución a cada grupo obtenido. Se procede, en un primer escenario, a incluir todas las variables que se han definido. Cabe recordar que el coeficiente de determinación obtenido para cada variable $\left(\mathrm{R}^{2}\right)$ da cuenta del grado de su contribución a la hora de definirse los doce grupos considerados.

Los resultados obtenidos para este primer escenario ofrecen valores elevados -superan $0,9 \mathrm{o}$ son muy próximos a dicho valor-, para las variables: índice de edificabilidad bruta, ocupación, densidad de alineación a vial y densidad de medianeras. No es el caso de la compacidad y la densidad de red viaria que registran los valores de 0,77 y 0,74 respectivamente. Como se ha visto en el análisis aislado de las visualizaciones correspondientes a cada variable por separado, la densidad de la red viaria ofrece una imagen más bien confusa. De hecho, la presencia de singularidades, tales como avenidas y otros elementos de la red básica, puede distorsionar los resultados, asignando paquetes de ciudad confróntate a determinados ejes a grupos específicos, cuando, en realidad, su forma urbana es análoga a sus tejidos contiguos. Se considera, pues, que en realidad el rol del viario puede ser obviado en este caso dado que ya estaría presente de forma indirecta a través de parámetros como la ocupación. El segundo escenario propuesto contempla todas las variables excepto la densidad de la red viaria. Los valores de $\mathrm{R}^{2}$ aumentan en todos los casos y se sitúan claramente entorno al valor 0,90 (véase tabla1).

Tabla 1. Contribución de las variables consideradas en cada escenario

\begin{tabular}{|l|c|c|}
\hline \multicolumn{1}{|c|}{ Variable } & $\begin{array}{c}\text { Escenario 1 } \\
\left(\mathbf{R}^{\mathbf{2}}\right)\end{array}$ & $\begin{array}{c}\text { Escenario 2 } \\
\left(\mathbf{R}^{\mathbf{2}}\right)\end{array}$ \\
\hline Edificabilidad bruta & 0,91 & 0,92 \\
\hline Ocupación & 0,87 & 0,90 \\
\hline Densidad alin. a vial & 0,88 & 0,90 \\
\hline Densidad medianeras & 0,90 & 0,93 \\
\hline Compacidad & 0,77 & 0,86 \\
\hline Densidad red viaria & 0,74 & - \\
\hline
\end{tabular}

Fte. Elaboración propia

Cabe recordar, en cualquier caso, que la validación de los resultados depende de la observación minuciosa de los grupos obtenidos, en el sentido de interpretar las tipologías que representan en cada caso. Para acometer dicho objetivo se visualizan los doce grupos obtenidos y se resumen los valores medios que toman las variables. Todo ello para proceder a bautizarlos manualmente en base a dichos valores y el sentido que toman una vez superpuestos con la cartografía convencional.

Con este procedimiento se propone la clasificación que se recoge en la tabla 2 y la figura 4 que representa los agrupamientos -análisis de clústeres- de la forma urbana. En primer lugar, se realiza una primera lectura que permite distinguir entre un grupo que denominaremos "histórico + compacto intenso", un conjunto de tejidos que agrupamos bajo el nombre de "Compactos" que se corresponden, generalmente, con las ordenaciones en manzana cerrada, un conjunto que denominaremos "manzana abierta" y las "bajas densidades".

El grupo "histórico + compacto intenso" es compatible con el casco antiguo, las agrupaciones suburbanas y otros tejidos compactos en los cuales se observa una ocupación muy 
Mercadé Aloy, J., Magrinyà Torner, F., Cervera Alonso de Medina, M. (2018): "Descifrando la forma urbana: un análisis de patrones de agrupamiento basado en SIG", GeoFocus (Artículos), no 22, p. 3-19. ISSN: $1578-5157$ http://dx.doi.org/10.21138/GF.612

elevada y una densidad de alineación a vial muy considerable -pocos espacios libres y mucho perímetro de fachada- en combinación con una densidad de medianeras muy importante -parcela pequeña- y un índice de edificabilidad considerable.

A continuación vienen los tejidos "compactos" que se organizan en tres categorías bien diferenciadas. Los "compactos 1 " son el grupo con un índice de edificabilidad más elevado y destacan, también, por registrar la compacidad muy elevada, así como una densidad de medianeras claramente inferior al grupo "histórico + compacto intenso". Se trata esencialmente del Ensanche de Barcelona. Los sucesivos "compactos" 2, 3 y 4 van describiendo otras realidades, generalmente en manzana cerrada, en la cuales la intensidad de techo va disminuyendo mientras que la densidad de alineación a vial propia de este tipo de tejidos se mantiene.

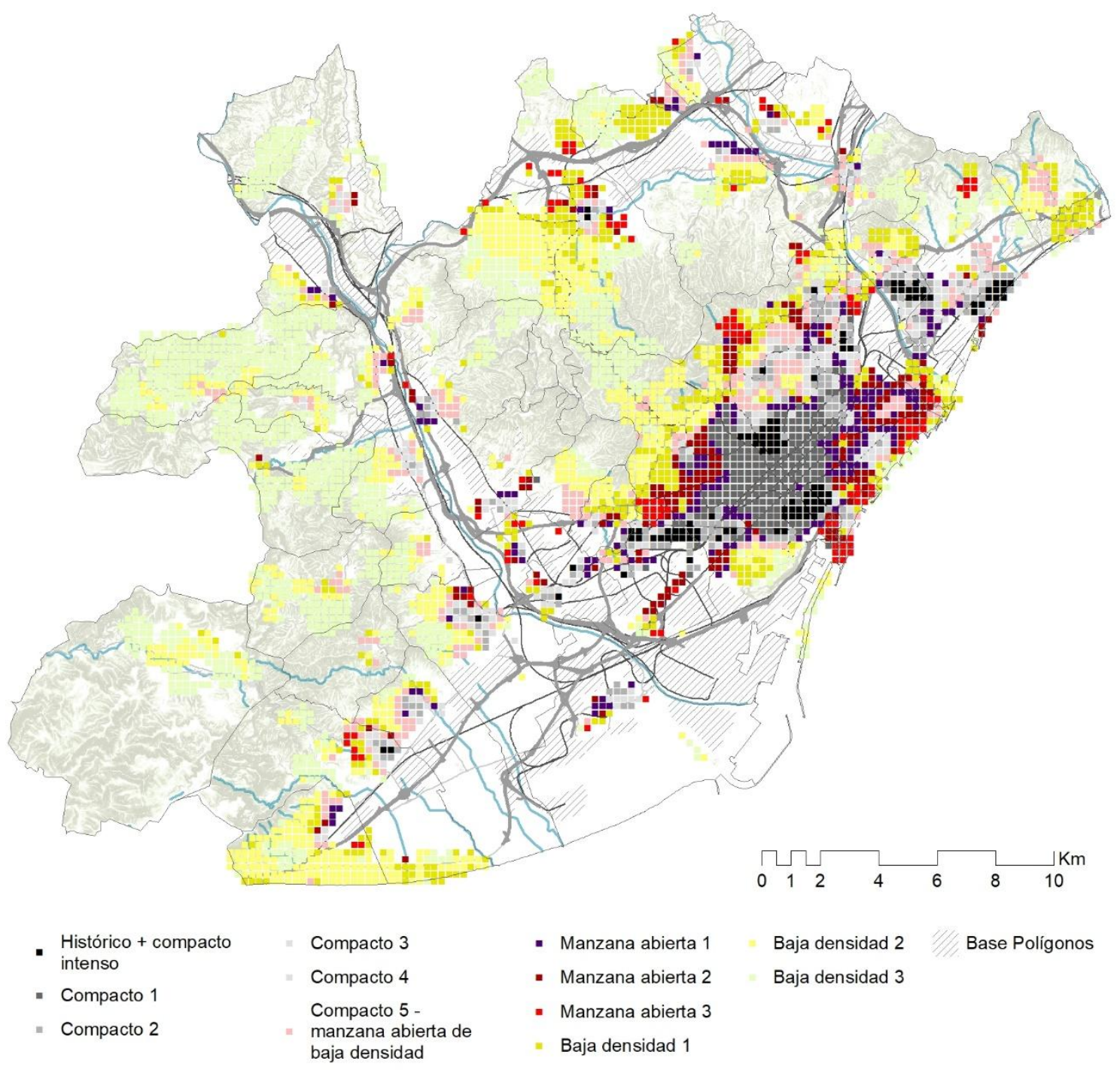

Figura 4. Establecimiento de grupos a partir del análisis de clústeres.

Fte.: Elaboración propia a partir de datos catastrales 2017. Dirección General del Catastro. 
Mercadé Aloy, J., Magrinyà Torner, F., Cervera Alonso de Medina, M. (2018): "Descifrando la forma urbana: un análisis de patrones de agrupamiento basado en SIG", GeoFocus (Artículos), no 22, p. 3-19. ISSN: $1578-5157$ http://dx.doi.org/10.21138/GF.612

Seguidamente, se considera el grupo "compacto $5+$ manzana abierta de baja densidad" que se corresponde con un conjunto híbrido, de transición, en el cual coexisten tejidos de alineación a vial con otros que presentan retranqueos en configuraciones espaciales resultantes con un índice de techo edificado bruto inferior a la unidad. Esta transición, abre las puertas a los conjuntos de ordenación en manzana abierta. En este caso, decaen inmediatamente los valores de densidad en alineación de vial y se abre un amplio abanico de valores de la edificabilidad, la ocupación y la compacidad. Se establecen tres agrupamientos en "manzana abierta" que experimentan valores de techo edificado decrecientes y compacidades crecientes.

Finalmente, se establecen tres paquetes de baja densidad cuya densidad en alineación de vial y de medianeras son prácticamente nula y muy reducida respectivamente. Los matices entre los tres subgrupos que se han considerado con los identificadores 1,2 y 3 radican en su índice de techo construido y compacidad decrecientes, que dan cuenta, en los extremos, de los tejidos que incorporan ciertos agrupamientos de viviendas más compactos hasta la edificación unifamiliar estrictamente aislada.

Tabla 2. Establecimiento de grupos y valores medios de las variables descriptivas

\begin{tabular}{|l|c|c|c|c|c|}
\hline \multicolumn{1}{|c|}{ Variable } & $\begin{array}{c}\text { Edif. } \\
\text { bruta }\end{array}$ & Ocupación & $\begin{array}{c}\text { Dens. alin. a } \\
\text { vial }\end{array}$ & $\begin{array}{c}\text { Dens. } \\
\text { medianeras }\end{array}$ & $\begin{array}{c}\text { Compaci } \\
\text { dad }\end{array}$ \\
\hline $\begin{array}{l}\text { Histórico + compacto } \\
\text { intenso }\end{array}$ & 2,315 & 0,582 & 0,043 & 0,178 & 2,093 \\
\hline Compactos 1 & 2,726 & 0,510 & 0,025 & 0,101 & 2,456 \\
\hline Compactos 2 & 1,830 & 0,478 & 0,034 & 0,127 & 2,064 \\
\hline Compactos 3 & 1,326 & 0,373 & 0,026 & 0,084 & 2,021 \\
\hline Compactos 4 & 0,805 & 0,554 & 0,033 & 0,019 & 1,291 \\
\hline $\begin{array}{l}\text { Compacto 5 + } \\
\text { manzana abierta de } \\
\text { baja densidad }\end{array}$ & 0,711 & 0,244 & 0,012 & 0,034 & 1,803 \\
\hline Manzana abierta 1 & 1,542 & 0,334 & 0,019 & 0,045 & 2,351 \\
\hline Manzana abierta 2 & 1,147 & 0,248 & 0,009 & 0,019 & 2,526 \\
\hline Manzana abierta 3 & 0,435 & 0,113 & 0,002 & 0,004 & 2,759 \\
\hline Baja densidad 1 & 0,350 & 0,120 & 0,002 & 0,005 & 1,963 \\
\hline Baja densidad 2 & 0,262 & 0,136 & 0,002 & 0,004 & 1,434 \\
\hline Baja densidad 3 & 0,095 & 0,062 & 0,001 & 0,001 & 1,150 \\
\hline
\end{tabular}

Fte.; Elaboración propia 
Mercadé Aloy, J., Magrinyà Torner, F., Cervera Alonso de Medina, M. (2018): "Descifrando la forma urbana: un análisis de patrones de agrupamiento basado en SIG", GeoFocus (Artículos), no 22, p. 3-19. ISSN: $1578-5157$ http://dx.doi.org/10.21138/GF.612

\section{Discusión y valoración de hallazgos}

Los clústeres de ciudad homogénea obtenidos se corresponden claramente con tejidos urbanos disimilares y reconocibles desde la disciplinas afines a los estudios de morfología urbana. Se revela un paisaje clasificado automáticamente en los grandes grupos de tejidos urbanos que se gradúan internamente mediante subgrupos.

El encaje de nuestro enfoque en el contexto de otros estudios anteriores obliga a centrarse en la elección de atributos relevantes y la definición de una unidad mínima para ser asignada a uno u otro grupo.

Por un lado, Gil et al. (2012) nos recuerdan que según Witten y Frank (2005) la mejor manera de elegir un conjunto de variables relevantes es manualmente en base al conocimiento del problema que se aborda y el significado de los atributos que intervienen. Continúan sugiriendo que no existe un conjunto de atributos erróneo, sino más bien un enfoque determinado que pudiera hacer más o menos énfasis en ciertas características: estructurales, geométricas, relacionales, físicas, estilísticas, históricas o socioculturales. Cabe destacar que nuestra aproximación concentra su enfoque en las variables que derivan de la forma urbana estricta -manzana, parcela y edificación-, renunciando a la topología de la red de calles de los paquetes de tejido urbano expresamente. Nos referimos, aquí, a las medidas de centralidad (para una revisión de las medidas de centralidad ver p.e. Mercadé, 2016) u otros conceptos relacionados con la accesibilidad y la topología de redes que se incluyen en parte de los estudios de referencia (ver p.e. Oliveira, 2012; Gil et al., 2012; Schrimer y Axhausen, 2015). En nuestro caso se desea obtener únicamente un conjunto de tejidos urbanos clasificados según su forma, en base a relaciones de estricta proximidad sin que el hecho de pertenecer o no a una determinada malla de calles de patrón reconocible -distritos, crecimientos o paquetes de ciudad planificadas en un periodo determinado- presuponga su inclusión en un determinado grupo. Es decir que se quiere prescindir de los patrones de red viaria y centrarse en el concepto de sistema de ordenación de manera graduada.

La propuesta opta por tomar variables clásicas como la edificabilidad y la ocupación e introducir atributos de densidad -densidad de alineación a vial y de medianeras- y de compacidad. Los valores de $\mathrm{R}^{2}$ en relación a las medidas de densidad propuestas son incluso superiores a la ocupación, siendo, pues, esta elección una contribución importante. La condición de fachada en alineación a vial da cuenta de dicho sistema de ordenación y de la densidad viaria implícitamente. La densidad de medianeras - parámetro más novedoso que se propone- da cuenta del tamaño de parcela, de la intensidad de los tejidos, en sistemas de ordenación en alineación a vial.

Por otro lado, debe remarcarse que la mayoría de estudios toman como unidad mínima los elementos desagregados de la morfología urbana - manzanas, parcelas, edificios y/o segmentos del grafo de calles- para la confección de clústeres y la visualización de resultados. Ello conlleva desventajas: la pérdida de noción de tejido en el establecimiento de grupos y la dificultad de visualizar los resultados a escala regional. El hecho de no tomar como muestra mínima un trozo de tejido que incluya manzanas y calles obliga a introducir parámetros adicionales que incluyan el entorno de espacios libres de edificación complicando innecesariamente el enfoque. 
Mercadé Aloy, J., Magrinyà Torner, F., Cervera Alonso de Medina, M. (2018): "Descifrando la forma urbana: un análisis de patrones de agrupamiento basado en SIG", GeoFocus (Artículos), no 22, p. 3-19. ISSN: $1578-5157$ http://dx.doi.org/10.21138/GF.612

\section{Conclusiones}

Los resultados obtenidos revelan un mosaico de tejidos urbanos comprensible y listo para servir como base para ulteriores estudios urbanos. La introducción de variables descriptivas de densidad -de alineación a vial y de medianeras-, menos usuales, contribuye notablemente a la confección de grupos nítidos con precisión. El método de captura de los valores que toman los atributos mediante círculos solapados permite atenuar las singularidades, ofreciendo una lectura continua en la cual interviene el tejido como tal -edificios, manzanas y calles conjuntamente.

La principal limitación detectada es la dificultad de definición de un radio adecuado de captura de geoinformación para cada escala de estudio. El valor adoptado debe ser suficientemente grande para incluir parte significativa de tejido en la muestra y suficientemente pequeño como para no capturar un exceso de tipologías de forma a la vez que pudieran distorsionar los resultados.

Finalmente quiere apuntarse que el método de captura de datos permite el tratamiento simultáneo de otro tipo de parámetros complementarios que intervienen en la forma sostenible usos, transporte público, salud, equidad, energía, calidad ambiental y configuración socioeconómica entre otros (ver p.e. Talen, 2011)- que pudieran relacionarse espacialmente con la clasificación de forma urbana propuesta. En el caso del Área Metropolitana de Barcelona es especialmente relevante mencionar el proceso actual de redacción del Plan Director Urbanístico metropolitano, que incluye múltiples jornadas técnicas de debate. Les reflexiones teóricas en torno al futuro proyecto de metabolismo urbano implicaran necesariamente relacionar forma urbana con fisiología.

\section{Referencias bibliográficas}

Alexander, C., Ishikawa, S., y Silverstein, M. (1977): A Pattern Language. Ch. Alexander. http://doi.org/10.2307/1574526

Área Metropolitana de Barcelona (n.d.): L'àrea metropolitana. http://www.amb.cat/s/web/areametropolitana/area-metropolitana.html (consultado 08-03-2018).

Berghauser Pont, M. Y., y Haupt, P. A. (2007): The relation between urban form and density. Urban Morphology, 11(1), 62-6.

Boeri, S., Lanzani, A., y Marini, E. (1993): Il Territorio che cambia: ambiente, paesaggi e immagini della regione milanese. Milano: AIM Associazione Interessi Metropolitani. Retrieved from http://cataleg.upc.edu/record=b1136007 S1*cat

Cardozo, O. D., Gutiérrez Puebla, J. y García Palomares, J. C. (2010): Influencia de la morfología urbana en la demanda de transporte público: análisis mediante SIG y modelos de regresión múltiple. Geofocus, 10, 82-102.

Solà-Morales, M. de (1979): Una cartografia per il territorio catalano / A cartography for the Catalan Territory. Lotus International, (23), 10-33.

Solà-Morales, M. de (1989): The culture of description. Perspecta, 16-25.

Solà-Morales, M. de (1993): Les Formes de creixement urbà. Barcelona : Edicions UPC. Retrieved from http://cataleg.upc.edu/record=b1082191 S1*cat 
Mercadé Aloy, J., Magrinyà Torner, F., Cervera Alonso de Medina, M. (2018): "Descifrando la forma urbana: un análisis de patrones de agrupamiento basado en SIG", GeoFocus (Artículos), no 22, p. 3-19. ISSN: $1578-5157$ http://dx.doi.org/10.21138/GF.612

Dillenburger, B. (2010): Space Index: A retrieval-system for building-plots. In 28th Conference on Education in Computer Aided Architectural Design in Europe (eCAADe 2010).

Esteban-Noguera, J. (1981): Elementos de ordenación urbana. Barcelona: Colegio Oficial de Arquitectos de Catalunya. Retrieved from http://cataleg.upc.edu/record=b1029509 S1*cat

ESRI How Grouping Halysis Horks. http://desktop.arcgis.com/en/arcmap/10.3/tools/spatial-statistics-toolbox/how-grouping-analysisworks.htm (consultado 07-12-2017)

Font Arellano, A. (2012): Patrons urbanistics de les activitats econòmiques : Regió Metropolitana de Barcelona = Urban patterns of economic activities: Barcelona Metropolitan Region. Barcelona: Generalitat de Catalunya. Retrieved from http://cataleg.upc.edu/record=b1417858 S1*cat

Font Arellano, A. (2004): L'Explosio de la ciutat $=$ The explosion of the city : morfologies, mirades $i$ mocions sobre les transformacions territorials recents en les regions urbanes de l'Europa Meridional: morphologies, observations and motions within recent territorial transforma. Barcelona: Fòrum Universal de les Cultures. Retrieved from http://cataleg.upc.edu/record=b1253660 S1*cat

Font Arellano, A., Llop, C., y Vilanova i Claret, J. M. (1999): La Construcció del territori metropolità : morfogènesi de la regió urbana de Barcelona. Barcelona: Àrea Metropolitana de Barcelona. Mancomunitat de Municipis. Retrieved from http://cataleg.upc.edu/record=b1162510 S1*cat

García, F. (2016): Compacidad y densidad de las ciudades Españolas. Eure, 42, 5-27. http://doi.org/10.4067/S0250-71612016000300001

Gil, J., Beirão, J. N., Montenegro, N., \& Duarte, J. P. (2012): On the discovery of urban typologies: Data mining the many dimensions of urban form. Urban Morphology, 16(1), 27-40.

Haggag, M. A., y Ayad, H. M. (2002): The urban structural units method: A basis for evaluating environmental prospects for sustainable development. Urban Design International, 7(2), 97-108. http://doi.org/10.1057/palgrave.udi.9000071

Hamaina, R., Leduc, T., y Moreau, G. (2012): Towards urban fabrics characterization based on buildings footprints. In Lecture Notes in Geoinformation and Cartography. http://doi.org/10.1007/978-3-642-29063-3_18

Hanson, J. (1998): Decoding homes and houses. Cambridge University Press.

Hecht, R., Herold, H., Meinel, G., y Buchroithner, M. (2013): Automatic Derivation of Urban Structure Types from Topographic Maps by Means of Image Analysis and Machine Learning. 26th International Cartographic Conference - Proceedings: International Cartographic Association, 2013.

Hillier, B. (1996): Space is the Machine: A Configurational Theory of Architecture (Cambridge University Press, Cambridge).

Hillier, B., y Hanson, J. (1984): The social logic of space. Cambridge university press. 
Mercadé Aloy, J., Magrinyà Torner, F., Cervera Alonso de Medina, M. (2018): "Descifrando la forma urbana: un análisis de patrones de agrupamiento basado en SIG", GeoFocus (Artículos), no 22, p. 3-19. ISSN: $1578-5157$ http://dx.doi.org/10.21138/GF.612

Jain, A. K. (2010): Data clustering: 50 years beyond K-means. Pattern Recognition Letters, 31, 651-666. http://doi.org/10.1016/j.patrec.2009.09.011

Jenks, G. F. (1967): The data model concept in statistical mapping. International Yearbook of Cartography, 7(1), 186-190.

Laskari, A., Hanna, S., y Derix, C. (2008): Urban identity through quantifiable spatial attributes Coherence and dispersion of local identity through the automated comparative analysis of building block plans. Design Computing and Cognition '08. http://doi.org/10.1007/978-1-4020-8728-8_32

Lloyd, S. P. (1982): Least Squares Quantization in PCM. IEEE Transactions on Information Theory. http://doi.org/10.1109/TIT.1982.1056489

Macqueen, J. (1967): Some methods for classification and analysis of multivariate observations. Proceedings of the Fifth Berkeley Symposium on Mathematical Statistics and Probability. http://doi.org/citeulike-article-id:6083430

Marshall, S. (2005): Urban Pattern Specification. Solutions, (January), 1-48.

Meinel, G., Hecht, R., Herold, H., Schiller, G., Spangenberg, M., Burgdorf, M., y Kawka, R. (2008): Automatische Ableitung von stadtstrukturellen Grundlagendaten und Integration in einem Geographischen Informationssystem. Forschungen.

Mercadé-Aloy, J. (2016): Taming the road: a GIS-based visualisation and spatial analysis methodology towards retrofitting the intermediate urban mosaic. TDX (Tesis Doctorals En Xarxa). Retrieved from http://www.tesisenred.net/handle/10803/371140

Morganti, M., Coch Roura, H., \& Cecere, C. (2012). The effect of urban obstructions in Mediterranean climates: Built form typology, density and energy. Architecture, City and Environment, 19, 13-26. http://doi.org/10.5821/ace.v7i19.2559

Moudon, A. V. (1997): Urban morphology as an emerging interdisciplinary field. Urban Morphology, 1, 3-10. http://doi.org/1027-4278

Oliveira, V. (2013): Morpho: A methodology for assessing urban form. Urban Morphology, 17(1), 149-161.

Schirmer, P. M., y Axhausen, K. W. (2015): A multiscale classification of urban morphology. Journal of Transport and Land Use, 9(1), 101-130. http://doi.org/10.5198/jtlu.2015.667

Secchi, B. (1993): Le trasformazioni dell'habitat urbano. Quaderno Della Ricerca Sulle Trasformazioni Dell'habitat Urbano in Europa, 1.

Sevtsuk, A., y Mekonnen, M. (2012): Urban network analysis. A new toolbox for ArcGIS. Revue Internationale de Géomatique. http://doi.org/10.3166/rig.22.287-305

Stead, D., y Marshall, S. (2001): The Relationships between Urban Form and Travel Patterns. An International Review and Evaluation. European Journal of Transport and Infrastructure Research, $1(2), 113-141$.

Steinhaus, H. (1956): Sur la division des corps matériels en parties. Bulletin de l'Académie Polonaise Des Sciences - Classe III. http://doi.org/citeulike-article-id:3738255 
Mercadé Aloy, J., Magrinyà Torner, F., Cervera Alonso de Medina, M. (2018): "Descifrando la forma urbana: un análisis de patrones de agrupamiento basado en SIG", GeoFocus (Artículos), no 22, p. 3-19. ISSN: $1578-5157$ http://dx.doi.org/10.21138/GF.612

Talen, E. (2011): Sprawl retrofit: Sustainable urban form in unsustainable places. Environment and Planning B: Planning and Design, 38(6), 952-978. http://doi.org/10.1068/b37048

Thomas, I., Frankhauser, P., Frenay, B., Verleysen, M., y Samos-Matisse, S. M. (2010): Clustering patterns of urban built-up areas with curves of fractal scaling behaviour. Environment and Planning B: Planning and Design. http://doi.org/10.1068/b36039

Van den Hoek, J. (2009): The Mixed Use Index (Mixed-use Index) as Planning Tool for (New) Towns in the 21st Century. New Towns for the 21st Century: The Planned vs, the Unplanned City. Amsterdam, NL: SUN Architecture.

Vilagrasa-Ibarz, J. (1998): The study of urban form in Spain. Urban Morphology, 2(1), 35-44.

Witten, I., \& Frank, E. F. (2005): Data Mining: Pratical Machine Learning Tools and Techiniques 2nd edition. Machine Learning. http://doi.org/10.1002/1521-3773(20010316)40:6<9823::AIDANIE9823>3.3.CO;2-C

Ye, Y., y Van Nes, A. (2013): Measuring urban maturation processes in Dutch and Chinese new towns: Combining street network configuration with building density and degree of land use diversification through GIS. The Journal of Space Syntax, 4(1), 18-37. 
